\title{
Daptomycin versus Vancomycin as Post-Operative Empirical Antibiotic Treatment for Prosthetic Joint Infections: A Case-Control Study
}

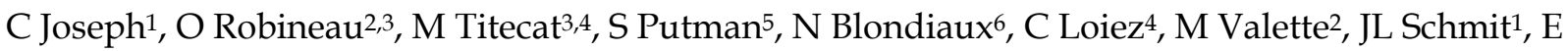 \\ Beltrand7, H Dézeque ${ }^{5}$, S Nguyen ${ }^{8}$, H Migaud ${ }^{3,5}$, E Senneville ${ }^{2,3,5}$ \\ 1. Infectious Diseases Department, University Hospital of Amiens, France \\ 2. Infectious Diseases Department, Gustave Dron Hospital of Tourcoing, France \\ 3. Faculty of Medicine of Lille, Lille University 2 \\ 4. Laboratory of Microbiology, University Hospital of Lille, France \\ 5. Orthopaedic Surgery Unit, University Hospital of Lille, France \\ 6. Laboratory of Microbiology, Gustave Dron Hospital of Tourcoing, France \\ 7. Orthopedic Surgery Unit, Gustave Dron Hospital, France \\ 8. General Hospital of Béthune \\ $\triangle$ Corresponding author: Eric Senneville, MD, PhD, ORCID Number : M-1832-2017, Infectious Diseases Department, Gustave Dron Hospital, 135 rue du \\ Président Coty, 59200 Tourcoing, France, French Referent Center for Osteo-Articular Infections (CRIOAC Lille-Tourcoing), Faculty of Medicine, Lille University \\ 2. Telephone: +33 320694 949; Fax: +33 320694 696; E-mail: esenneville@ch-tourcoing.fr \\ (C) Ivyspring International Publisher. This is an open access article distributed under the terms of the Creative Commons Attribution (CC BY-NC) license \\ (https://creativecommons.org/licenses/by-nc/4.0/). See http://ivyspring.com/terms for full terms and conditions.
}

Received: 2017.07.21; Accepted: 2018.06.16; Published: 2019.03.02

\begin{abstract}
Purpose: To compare safety and efficacy of Vancomycin (Van) versus Daptomycin (Dap) as post-operative empirical antibiotic treatment (PEAT) in patients with periprosthetic joint infections (PJIs).

Methods: Medical charts of patients treated empirically with Van or Dap in the post-operative period of total hip/knee prosthesis septic revision until the results of intra-operative culture were reviewed. Cefotaxime, cefepime or aztreonam were used in combination with Dap or Van.

Results: Twenty Dap patients were matched with 20 other Van patients according to the age and type of prosthesis. The ASA score and the distribution of the pathogens was similar in the two groups especially regarding the number of methicillin-resistant staphylococci. The mean duration of the PEAT was $6.07 \pm$ 0.85 days. A total of 17 episodes of adverse events (AE) in 10 patients $(25 \%)$ were recorded during the PEAT which led to discontinue the treatment in 5 patients, all of them treated with $\operatorname{Van}(P=0.02)$. At the end of a mean post-treatment follow-up of 618 +/- 219 days, 36 patients remained in remission of infection; 2 patients failed in each group.

Conclusions: Our observations suggest that PEAT with Van for septic revision of PJls is associated with a higher discontinuation rate due to AE but with a similar outcome than it is with Dap.
\end{abstract}

Key words: Daptomycin, Vancomycin, empirical antibiotic treatment, prosthetic joint infection

\section{Introduction}

Periprosthetic joint infections (PJIs) are treated in most cases by debridement, antibiotic therapy and implant retention (DAIR) or replacement in one or two-stage of the infected implants [1]. Given the important role of staphylococci in PJIs it seems important that the per-operative empirical antibiotic therapy (PEAT) would cover these pathogens including methicillin-resistant (MR) strains while waiting for the culture results of the intraoperative samples [2]. Although Gram-negative rods are less frequently encountered in this setting, they should also be covered by PEAT as well [3]. In our institution, Vancomycin (Van) is used as part of the PEAT in patients with PJI operated for DAIR and one/two- 
step exchange [2]. Dap is more effective than Van for the treatment of experimental foreign-body and systemic infections by biofilm-producing methicillin-resistant $S$. epidermidis [4,5]. The potential caveats of Dap are its cost and the selection of bacterial resistance.

The aim of the present retrospective study was to compare Van and Dap regarding both tolerability and efficacy in patients operated for PJIs.

\section{Material and Methods}

This was a retrospective study conducted at the French Northern-West referral center for osteoarticular infection between 01-2008 and 03-2013. From 01-2008 to 03-2012, Van was used as the first-line antibiotic agent in combination with a broad-spectrum $\beta$-lactam agent for PEAT in patients who underwent a septic revision for a PJI. Since 2012, we have replaced Van by Dap but neither the choice of $\beta$-lactam agent of the PEAT protocol nor the surgical procedures were modified in the meantime. We identified the patients who were given Dap as PEAT from 04-2012 until 03-2013 to assess their outcome at least one year after the end of the treatment and randomly included the same number of patients treated with Van selected on the patient's age and the type of prosthesis.

PJI was defined according to the Infectious Diseases Society of America (IDSA) guidelines criteria of PJI [1]. In this study, we defined acute PJIs as those with $\leq 2$ weeks of symptoms.

Remission was defined as the absence of local or systemic signs of PJI at the last contact and the absence of any new surgery or antibiotic therapy related to the PJI assessed $\geq 1$ year after the end of antibiotic treatment. Treatment failure was defined as any other outcome, including patient death related to the PJI.).

PEAT was administered intravenously (IV) immediately after all of the samples were taken. Cefotaxime (100mg/ $\mathrm{kg}$ IV divided into 3 daily doses), cefepime ( $2 \mathrm{~g}$ IV/6 to $8 \mathrm{~h}$ ) or aztreonam ( $2 \mathrm{~g}$ IV $/ 8 \mathrm{~h}$ ) were used in combination with Dap or Van in every patient. Van was administered IV with a loading dose of $15 \mathrm{mg} / \mathrm{kg}$ followed by a continuous infusion of a dose of 30 to $40 \mathrm{mg} / \mathrm{kg}$ per $24 \mathrm{~h}$. Serum levels of Van were monitored each 48 hours and daily doses were adapted to maintain serum trough levels of 20 to 30 $\mathrm{mg} / \mathrm{L}$. Dap was administered at a daily dose of 10 to $12 \mathrm{mg} / \mathrm{kg}$ in a single daily IV infusion over $30 \mathrm{~min}$ [6].

PEAT was continued until perioperatively obtained sample culture results were available, and then modified accordingly. Antibiotics were selected based on the patient's comorbidities and prescribed at doses adapted from those proposed by Zimmerli et al. [7]. After discharge from the hospital, the patient was followed up by both the referring surgeon and the infectious disease consultant 1 month after discharge, at the end of antibiotic treatment and last contact. The total duration of antimicrobial therapy was 3-6 months, IDSA [1].

We collected antibiotic regimen, doses, antibiotic treatment duration, and clinical and biological tolerance (side effects) under treatment. Nephrotoxicity was defined according to the Acute Kidney Injury Network classification as an acute (within 48h) decrease in kidney function, specified as an increase in serum creatinine of $\geq 0.3 \mathrm{mg} / \mathrm{dL}$, and/or an increase in $-50 \%$ of baseline serum creatinine [8]. Adverse events (AE) following Dap or Van was assessed according to the chronology of events, the need for reducing the daily dosage of the incriminated antibiotic, the data from any attempt to re-introduce the incriminated treatment and the type of recorded toxicity.

All patients' collected data were anonymized and recorded on a standardized form preventing any personal identification according to procedures defined by the French information protection commission (Commission Nationale de l'Informatique et des Libertés-CNIL); approval from the Institutional Review Board of our hospital was obtained ( $\mathrm{N}^{\circ}$ 2016/02).

\section{Results}

A total of 40 patients with PJI were analyzed, 20 who received Dap and 20 who received Van in the PEAT. The demographic data of the patients are reported in Table 1. Overall, the mean age (SD) was $66.6 \pm 14.6$ years and $45 \%$ were males. Patients of the two groups only differed on the type of surgery with a higher proportion of patients treated with DAIR in the Dap group (Table 1). There was a trend toward a higher serum C-reactive protein value in patients treated with Van (Table 1). All patients had a normal baseline serum creatinine concentration. The distribution of the pathogens was similar in the two especially regarding the number of MR-staphylococci and polymicrobial infections (Table 2). All Gram positive cocci identified in intraoperative samples had a MIC $\leq 1 \mathrm{mg} / \mathrm{L}$ for Van and $\leq 0.25 \mathrm{mg} / \mathrm{L}$ for Dap. The mean duration of the PEAT was $6.07 \pm 0.85$ days. The mean daily dose $( \pm$ SD, mg) of Dap and Van was $730.0 \pm 176.5$ and $3,252 \pm 1,077$, respectively, corresponding in $\mathrm{mg} / \mathrm{kg}$ to respectively $9.57 \pm 1.24$ and $33.05 \pm 14.83$. The duration of Van and Dap PEAT was $6.25 \pm 2.51$ and $4.40 \pm 2.82$, respectively. A total of 17 episodes of AEs in 10 patients (25\%) were recorded during the PEAT which led to discontinue the treatment in 5 patients $(12.5 \%)$, all treated with Van $(\mathrm{P}=0.02)$ (Table 3). Van therapy was discontinued after a mean duration of $3.4 \pm 1.1$ days. The reasons for discontinuing Van therapy were the occurrence of an acute renal insufficiency $(n=2)$ and thrombophlebitis 
$(\mathrm{n}=3)$. Patients who had to stop Van were older than the other Van patients $(75.7 \pm 8.04$ versus $64.1 \pm 13.6$ years 9 ; $p=0.065$ ) whereas creatinine blood concentration and clearance at baseline, concomitant use of nephrotoxic agents and diabetes were comparable. Oral linezolid was used to replace Van.

Culture-guided antibiotic regimens were not significantly different between Van and Dap groups in terms of both antibiotic agents and duration (Table 1). The mean follow-up of the 36 patients in remission was $618+/-219$ days. Four patients (2 in the Van group and 2 in the Dap group) patients failed due to Coagulase-negative Staphylococci (all susceptible to both Van and Dap). Three out of these 4 patients had been treated with DAIR and one with one-stage replacement; the mean delay between septic revision and diagnosis of infection was $65.8+/-22.9$ days (range 35-99). One patient in the Dap group died of a reason unrelated to the PJI.

Table 1. Compared characteristics and outcome of 40 patients treated empirically with either Vancomycin or Daptomycin for a periprosthetic joint infection

\begin{tabular}{|c|c|c|c|}
\hline & $\begin{array}{l}\text { Daptomycin } \\
\mathrm{n}=20(\%)\end{array}$ & $\begin{array}{l}\text { Vancomycin } \\
\mathrm{n}=\mathbf{2 0}(\%)\end{array}$ & $\mathbf{P}$ \\
\hline Age, years mean $\pm S D$ & $65.6 \pm 15.8$ & $67.5 \pm 13.1$ & .67 \\
\hline Male & $10(50)$ & $8(40)$ & .52 \\
\hline Body weight $\mathrm{kg}$, mean $\pm \mathrm{SD}$ & $77.5 \pm 20.4$ & $77.9 \pm 16.6$ & .9 \\
\hline Co-morbidity & $3^{*}(15)$ & $4^{* *}(20)$ & .9 \\
\hline Acute PJI & $9(45)$ & $9(45)$ & 1 \\
\hline Total hip arthroplasty & $16(80)$ & $16(80)$ & 1 \\
\hline Total knee arthroplasty & $4(20)$ & $4(20)$ & 1 \\
\hline Debridement-retention & $8(40)$ & $12(60)$ & 0.20 \\
\hline One-stage exchange & $8(40)$ & $6(30)$ & 0.51 \\
\hline Two-stage exchange & $4(20)$ & $2(10)$ & .38 \\
\hline $\begin{array}{l}\text { Time from incision to first } \\
\text { administration min, mean } \pm S D\end{array}$ & $94.1 \pm 36.7$ & $101.1 \pm 149.6$ & .59 \\
\hline $\begin{array}{l}\text { Baseline serum creatinine } \\
\text { concentration } \mathrm{mg} / \mathrm{L} \text {, mean } \pm \mathrm{SD}\end{array}$ & $6.56 \pm 1.74$ & $6.06 \pm 1.81$ & .52 \\
\hline C-reactive protein $\mathrm{mg} / \mathrm{L}$, mean $\pm \mathrm{SD}$ & $32.8 \pm 45.8$ & $70.6 \pm 68.8$ & 0.09 \\
\hline Combined antibiotics & & & .34 \\
\hline - cefotaxime & $10(50)$ & $11(55)$ & .75 \\
\hline - cefepime & $8(40)$ & $6(30)$ & .51 \\
\hline - aztreonam & $1(5)$ & 0 & .31 \\
\hline - gentamicin & $1(5)$ & $1(5)$ & 1 \\
\hline - ciprofloxacin & 0 & $2(10)$ & .15 \\
\hline Hospital stay length days, mean \pm SD & $22.1 \pm 23.4$ & $21.8 \pm 15.9$ & .59 \\
\hline \multicolumn{4}{|l|}{ Culture-guided antibiotic therapy } \\
\hline - mean duration, days \pm SD & $91.1 \pm 40.5$ & $102.9 \pm 86.2$ & 0.10 \\
\hline - rifampicin-levofloxacin & $9(45 \%)$ & $8(40 \%)$ & .75 \\
\hline - rifampicin-doxycyclin & $2(10 \%)$ & $2(10 \%)$ & 1 \\
\hline - rifampicin-cotrimoxazole & $1(5 \%)$ & $2(10 \%)$ & .55 \\
\hline - rifampicin-linezolid & $3(15 \%)$ & $2(10 \%)$ & .23 \\
\hline - clindamycin-levofloxacin & $1(5 \%)$ & $1(5 \%)$ & 1 \\
\hline - clindamycin-doxycyclin & $2(10 \%)$ & $2(10 \%)$ & 1 \\
\hline - ceftriaxone-levofloxacin & $2(10 \%)$ & $3(15 \%)$ & .23 \\
\hline \multicolumn{4}{|l|}{ Outcome } \\
\hline - death $* * *$ & $1(5 \%)$ & 0 & .31 \\
\hline - relapsing infection & $2(10 \%)$ & $2(10 \%)$ & 1 \\
\hline - remission & $17(85 \%)$ & $18(90 \%)$ & .63 \\
\hline
\end{tabular}

Table 2. Microbiological results of intraoperative samples culture in 40 patients treated empirically with either Daptomycin or Vancomycin

\begin{tabular}{llll}
\hline Microbiology & $\begin{array}{l}\text { Daptomycin } \\
\mathbf{N}^{\circ} \text { of strains (\% of } \\
\text { the total) }\end{array}$ & $\begin{array}{l}\text { Vancomycin } \\
\mathbf{N}^{\circ} \text { of strains (\% of } \\
\text { the total) }\end{array}$ & P \\
\hline $\begin{array}{l}\text { Gram-positive cocci } \\
\text { - Staphylococcus aureus }\end{array}$ & $23(92)$ & $20(74.1)$ & .09 \\
- Coagulase negative & $14(56)$ & $9(33.3)$ & .15 \\
staphylococci** & & $5(18.5)$ & .005 \\
- Corynebacterium spp. & $2(8)$ & 0 & .13 \\
- Enterococcus faecalis & $3(12)$ & $1(3.7)$ & .26 \\
- Propionibacterium acnes & 0 & $2(7.4)$ & .17 \\
- Streptococcus spp. & 0 & $3(11.1)$ & .09 \\
Gram-negative bacilli & $2(8)$ & $6(22.2)$ & .16 \\
- Escherichia coli & 0 & $4(14.0)$ & .05 \\
- Morganella morganii & $1(4)$ & 0 & .29 \\
- Proteus mirabilis & $1(4)$ & $2(7.4)$ & .60 \\
Obligate anaerobes & 0 & $1(3.7)$ & .33 \\
Total & $25(100)$ & $27(100)$ & .61 \\
Polymicrobial infections & $5(25)$ & $7(35)$ & \\
(No of cases) & & & \\
\hline * : all methicillin-susceptible strains, ** : including 15 methicillin-resistant strains $(9$ \\
in the Dap group and 6 in the Van group)
\end{tabular}

Table 3. Compared tolerance to antibiotic treatment of 40 patients treated empirically for either Vancomycin or Daptomycin

\begin{tabular}{|c|c|c|c|}
\hline AE episodes & $\begin{array}{l}\text { Daptomycin } \\
(n=20)\end{array}$ & $\begin{array}{l}\text { Vancomycin } \\
(n=20)\end{array}$ & $\mathbf{P}$ \\
\hline Allergy & 0 & $1(5 \%)$ & .31 \\
\hline Thrombophlebitis at the injection site & 0 & $2(10 \%)$ & .15 \\
\hline Nausea & $4(20 \%)$ & $4(20 \%)$ & 1 \\
\hline Diarrhoea & $2(10 \%)$ & $1(5 \%)$ & .54 \\
\hline Acute renal failure & 0 & $2(10 \%)$ & .15 \\
\hline Myalgia* & $1(5 \%)$ & 0 & .31 \\
\hline Total $\mathrm{N}^{\circ}$ of episodes of adverse events & $7(35 \%)$ & $10(50 \%)$ & .92 \\
\hline $\begin{array}{l}\text { Total } \mathrm{N}^{\circ} \text { of patients who experienced } \\
\text { adverse events }\end{array}$ & $4(20 \%)$ & $6(30 \%)$ & .47 \\
\hline $\begin{array}{l}\text { Total } \mathrm{N}^{\circ} \text { of patients with } \\
\text { discontinuations for adverse events }\end{array}$ & 0 & $5^{* *}(25 \%)$ & .02 \\
\hline
\end{tabular}

\section{Discussion and Conclusion}

We retrospectively compared 'empiric' postoperative treatment of Van with that of Dap in patients with PJIs. We found that the use of Dap as empirical coverage of Gram positive cocci in combination with $\beta$-lactam agent is associated with a better tolerance than that of Van. It was not associated with a better outcome assessed one year after the completion of treatment. In Ingram et al.'s study, $15.7 \%$ of the patients treated for mainly osteo-articular infections with Van developed nephrotoxicity which is slightly higher than in our series $(10 \%)$. The duration of exposition to Van treatment was significantly shorter in that study than it was in our patients [9]. Given the well-known risk factors for Van-related renal failure, it might be efficient in a cost effective approach to reserve PEAT with Dap to selected patients with higher Van maximum trough levels, longer duration of Van treatment, concomitant use of other nephro- 
toxic agents, critical illness or have a previously compromised renal function [10].

Our study has limitations. Firstly, the retrospective design of our study may have contributed to bias in selection of patients included. Secondly, AEs were attributed to Dap or Van, although we cannot be $100 \%$ certain about the causes of the AEs in our patients, but we feel that all necessary measures were taken to avoid erroneous evaluations. Thirdly, the absolute number of included patient was small, and the proportion numbers of SAE should be interpreted with caution. Fourthly, the influence of PEAT on the outcome may be not crucial, given the broad Gram-positive coverage of Dap and Van, and the directed antibiotic therapy for 3 to 6 months. Finally, the minimum post-treatment follow-up was only 12 months which does not allow to us affirm further infectious events did not occur later. In conclusion, our experience suggests that PEAT with Van for septic revision of PJIs is associated with a higher discontinuation rate due to AEs but with a similar outcome than it is with Dap. Larger size studies are warranted to confirm these preliminary data.

\section{Acknowledgments}

Thanks to Mr Philippe Choisy and Mr Marc Digumber for their technical assistance.

\section{Competing Interests}

ES has received grants (meeting supports and speaker honoraria) from NOVARTIS SA. The other authors have declared that no competing interest exists.

\section{References}

1. Osmon DR, Berbari EF, Berendt AR, et al.; Infectious Diseases Society of America.. Executive summary: diagnosis and management of prosthetic joint infection: clinical practice guidelines by the Infectious Diseases Society of America. Clin Infect Dis. 2013; 56: 1-10.

2. Senneville E, Joulie D, Legout L, et al. Outcome and predictors of treatment failure in total hip/knee prosthetic joint infections due to Staphylococcus aureus. Clin Infect Dis. 2011; 53: 334-40.

3. Titécat $M$, Senneville $E$, Wallet $F$, Dezèque $H$, Migaud $H$, Courcol RJ, Loïez C. Microbiologic profile of Staphylococci isolated from osteoarticular infections: evolution over ten years. Surg Infect (Larchmt). 2015; 16: 77-83

4. Domínguez-Herrera J, Docobo-Pérez F, López-Rojas R, et al. Efficacy of daptomycin versus vancomycin in an experimental model of foreign-body and systemic infection caused by biofilm producers and methicillin-resistant Staphylococcus epidermidis. Antimicrob Agents Chemother. 2012; 56: 613-7.

5. Murillo O, Garrigos C, Pachon ME, et al. Efficacy of high doses of daptomycin versus alternative therapies against experimental foreign-body infection by methicillin-resistant Staphylococcus aureus. Antimicrob. Agents Chemother. 2009; 53: 4252-7.

6. Senneville E, Caillon J, Calvet B, et al. Towards a definition of daptomycin optimal dose: Lessons learned from experimental and clinical data. Int J Antimicrob Agents. 2016; 47: 12-9.

7. Zimmerli W, Trampuz A, Ochsner PE. Prosthetic-joint infections. N Engl J Med. 2004;351:1645

8. Mehta RL, Kellum JA, Shah SV, et al.; Acute Kidney Injury Network. Acute Kidney Injury Network: report of an initiative to improve outcomes in acute kidney injury. Crit Care. 2007; 11: R31.

9. Ingram PR, Lye DC, Tambyah PA et al. Risk factors for nephrotoxicity associated with continuous vancomycin infusion in outpatient parenteral antibiotic therapy. J Antimicrob Chemother. 2008; 62: 168-71.
10. Vandecasteele SJ, De Vriese AS. Recent changes in vancomycin use in renal failure. Kidney International. 2010; 77: 760-4. 